\title{
Radial oscillations and the ion hose instability of an electron beam propagating in a periodic ion channel
}

\author{
R. A. Bosch and R. M. Gilgenbach \\ Intense Energy Beam Interaction Laboratory, Nuclear Engineering Department, University of Michigan, \\ Ann Arbor, Michigan 48109-2104
}

(Received 3 September 1987; accepted 24 November 1987)

\begin{abstract}
Recently, the propagation of an electron beam through an ion channel with periodically varying ion density has been proposed as a method of transporting or modulating an electron beam. A theoretical treatment indicates that, in the absence of an external magnetic field, cylindrically symmetric radial oscillations of the beam electrons are excited by the channel, with resonant excitation occurring when the wavelength of the ion density variation equals the betatron wavelength. An analysis of the ion focusing regime (IFR) ion hose instability indicates that the $e$-folding length of this instability increases when the variations of the electron density are increased. In the absence of an external magnetic field and damping, moderate electron density variations (caused, for example, by a periodic ion channel or the nonrigidity of the beam and channel) result in a wavelength and $e$-folding length on the order of the beam-averaged betatron wavelength.
\end{abstract}

\section{INTRODUCTION}

Propagation of an electron beam in an ion channel in the ion focusing regime (IFR) allows the beam to propagate without expanding from space-charge repulsion. In the IFR, the beam electrons undergo radial oscillations at a characteristic frequency, so that in the laboratory the beam diameter varies periodically with a wavelength known as the betatron wavelength. ${ }^{1}$ One of the fundamental physical limitations of IFR $e$-beam propagation has been the ion hose (ion resonance) instability, in which transverse oscillations of the $e$ beam and ion channel couple and grow.

Recently, the use of ion channels with a periodic variation in ion density has been proposed as a possible method of transporting and inducing oscillations in a propagating $e$ beam. ${ }^{2,3}$ Such oscillations may have application to free-electron laser wigglers or plasma wave particle accelerators. ${ }^{4}$ Miller and Gilgenbach ${ }^{3}$ have performed analytic and numerical calculations describing the propagation of $e$ beams in channels where the ion density vanished periodically, observing stable transport with oscillations in the beam envelope at the betatron wavelength as well as the wavelength of the ion density variation.

In this paper, we derive an analytic solution for the radial motion of an arbitrary fluid element of a cylindrically symmetric electron beam propagating in a previously ionized channel for which the ion density profile varies sinusoidally with axial position. It is assumed that the ions are stationary and that the electron beam instantaneously ejects radially the plasma electrons associated with these ions. We perform the analysis in the frame of reference moving with the $e$ beam. The results, given in Sec. II, indicate that resonant excitation of cylindrically symmetric e-beam oscillations occurs when the wavelength of the ion density variation equals the betatron wavelength of the $e$-beam electrons. In Sec. III, we present calculations of the $e$-folding length and wavelength for the ion hose instability in a periodic ion channel. The calculations show that a periodic ion channel can result in an increase in the wavelength and $e$ folding length of the instability.

\section{OSCILLATIONS IN ELECTRON BEAM RADIUS}

\section{A. Basic equations and assumptions}

Let us consider an $e$ beam propagating in the $z$ direction in a periodic ion density channel. We assume a nonrotating beam with cylindrical symmetry and we perform the calculations in a frame of reference moving with the electron beam. At a fixed axial position in this frame, the periodic ion density channel appears as a time-varying ion density:

$$
n_{i}(r, t)=n_{0}(r)+n_{1}(r) \cos \omega t .
$$

(In the lab frame,

$$
n_{i_{\mathrm{lab}}}(r, z)=(1 / \gamma)\left[n_{0}(r)+n_{1}(r) \cos (2 \pi z / \lambda)\right],
$$

where $\lambda=2 \pi \gamma \beta c / \omega, \beta=v / c$, and $\gamma=\left(1-\beta^{2}\right)^{-1 / 2}$ describe the $e$-beam velocity.) We consider ion density variations with wavelengths long compared to the $e$-beam radius, so that the component of electric field in the $z$ direction is ignorable. ${ }^{3}$ At the radial position $r$, the radial electric field resulting from the ion charge is

$$
E_{i}(r, t)=\frac{\int_{0}^{r} e\left[n_{0}\left(r^{\prime}\right)+n_{1}\left(r^{\prime}\right) \cos \omega t\right] 2 \pi r^{\prime} d r^{\prime}}{\epsilon_{0}(2 \pi r)}
$$

Letting $n_{e}(r, t)$ equal the electron density, the radial electric field at position $r$ resulting from the electron charge is

$$
E_{e}(r, t)=\frac{\int_{0}^{r}-e n_{e}\left(r^{\prime}, t\right) 2 \pi r^{\prime} d r^{\prime}}{\epsilon_{0}(2 \pi r)} .
$$

Using Eqs. (2) and (3), we write the equation of motion for a fluid element of electrons: 


$$
\begin{aligned}
\frac{d^{2} r}{d t^{2}}= & -\frac{e E}{m_{e}}-\frac{k T_{e}}{m_{e} n_{e}} \frac{d n_{e}}{d r} \\
= & \frac{\int_{0}^{r} e^{2} n_{e}\left(r^{\prime}, t\right) 2 \pi r^{\prime} d r^{\prime}}{m_{e} \epsilon_{0}(2 \pi r)} \\
& -\frac{\int_{0}^{r} e^{2}\left[n_{0}\left(r^{\prime}\right)+n_{1}\left(r^{\prime}\right) \cos \omega t\right] 2 \pi r^{\prime} d r^{\prime}}{m_{e} \epsilon_{0}(2 \pi r)} \\
& -\frac{k T_{e}}{m_{e} n_{e}} \frac{d n_{e}}{d r} .
\end{aligned}
$$

In this equation, we ignore the magnetic forces that result from the ion current that is present in the electron frame of reference. This is reasonable since the electron velocities are low in this frame. For a relativistic $e$ beam, this requires that the beam current (in the lab frame) be small compared to the Alfén current. ${ }^{5}$ This is discussed further in the Appendix. For the case of $e$-beam propagation parallel to a weak magnetic field $B_{0}$, the magnetic forces can be neglected in Eq. (4) provided that the electron cyclotron frequency $\left(e B_{0} / m_{e}\right)$ is much smaller than $\omega$ and $\omega_{p e}$ (the electron plasma frequency measured in the frame of reference moving with the $e$ beam). The temperature $T_{e}$ corresponds to an emittance in the laboratory frame.

We note that the electron charge $-\int_{0}^{r(t)} e n_{e}\left(r^{\prime}, t\right)$ $\times 2 \pi r^{\prime} d r^{\prime}$ is a constant of the motion. As a result, the motion of an arbitrary fluid element in Eq. (4) is independent of the motion of the other fluid elements when $T_{e}=0$, while the pressure gradient term couples neighboring fluid elements when $T_{e} \neq 0$.

For the case where $n_{1}(r) \equiv 0$ and $T_{e}=0$, Eq. (4) indicates that a force-free equilibrium exists when $n_{e}(r, t)$ $=n_{0}(r)$. At the other extreme, the pressure gradient force will dominate the electron electric force, $e E_{e}(r)$, in a hot beam, where the electron Debye length, $\lambda_{\mathrm{D} e}$, exceeds the ion channel radius. In this case, the equilibrium electron distribution is a Maxwellian, $n_{e}(r, t)=n_{\infty} \exp \left[e \phi(r) / k T_{e}\right]$, where $\phi(r)$ is the electrostatic potential resulting from the ion channel. This situation corresponds to the case where the magnetic and electric fields from the $e$ beam are ignorable in the laboratory frame since they cancel to order $1 / \gamma^{2}$.

For a cool beam, the pressure gradient term is a small correction in regions where the electron Debye length is much smaller than the beam radius. Thus we can substitute $n_{0}(r)$ for $n_{e}(r, t)$ in the pressure gradient term in order to estimate its value at equilibrium. With a Gaussian ion channel profile $\left[n_{0}(r)=n_{0}(0) \exp \left(-r^{2} / r_{c}^{2}\right)\right]$, the inclusion of the pressure gradient term changes the equilibrium condition to

$$
n_{e}(r, t)=n_{0}(r)-4 \epsilon_{0} k T_{e} / e^{2} r_{c}^{2}
$$

in those regions of the beam where $\lambda_{\mathrm{De}} \ll r_{c}$.

Consider a perturbation about the force-free equilibrium. Let $r_{0}$ equal the equilibrium position of an arbitrary fluid element, and define $\delta(t) \equiv r(t)-r_{0}$ to be the displacement of this fluid element from equilibrium. For small displacements such that $\delta \& r_{0}$, we can reduce Eq. (4) by utilizing the fact that $\int_{0}^{r(t)} n_{e}\left(r^{\prime}, t\right) 2 \pi r^{\prime} d r^{\prime}$ is constant. We assume that the electron density gradient term for a given fluid element, $\left(1 / n_{e}\right)\left[d n_{e}(r, t) / d r\right]$, is inversely proportional to $r(t)$, as it would be if the $e$ beam contracted and expanded without changing shape. We let $T_{e}(r(t)) \propto[r(t)]^{-2\left(c_{P} / c_{V}-1\right)}$, which describes adiabatic contractions and expansions of the beam $\left(c_{P} / c_{V}=1\right.$ describes the isothermal case $)$. This results in the linear equation

$$
\begin{aligned}
\frac{d^{2} \delta}{d t^{2}}= & -\left.\delta\left(\frac{e^{2} n_{0}}{\epsilon_{0} m_{e}}-\frac{k T_{e}}{m n_{e}} \frac{d n_{e}}{d r} \frac{2\left[\left(c_{P} / c_{V}\right)-1\right]}{r}\right)\right|_{r_{0}} \\
& -\frac{\int_{0}^{r_{0}} e^{2}\left[n_{1}\left(r^{\prime}\right) \cos \omega t\right] 2 \pi r^{\prime} d r^{\prime}}{2 \pi \epsilon_{0} m_{e} r_{0}}
\end{aligned}
$$

This equation describes a driven harmonic oscillator with solution

$$
\begin{aligned}
r(t)= & r_{0}+a_{0} \cos \left(\omega_{0} t+\theta\right) \\
& +\frac{e^{2} \int_{0}^{r_{0}} n_{1}\left(r^{\prime}\right) 2 \pi r^{\prime} d r^{\prime}}{2 \pi \epsilon_{0} m_{e} r_{0}}\left(\frac{1}{\omega^{2}-\omega_{0}^{2}}\right) \cos \omega t,
\end{aligned}
$$

where

$\omega_{0}^{2}=\left.\left(\frac{e^{2} n_{0}}{\epsilon_{0} m_{e}}-\frac{k T_{e}}{m n_{e}} \frac{d n_{e}}{d r} \frac{2\left[\left(c_{P} / c_{V}\right)-1\right]}{r}\right)\right|_{r_{0}}$.

For a cool beam in a Gaussian ion channel with $\lambda_{\mathrm{D} e} \ll r_{c}$, we can write Eq. (7) in terms of the local electron plasma frequency $\left(\omega_{p e}=\left[n_{e}\left(r_{0}\right) e^{2} / \epsilon_{0} m_{e}\right]^{1 / 2}\right)$, obtaining

$$
\omega_{0}^{2}=\omega_{p e}^{2}+\left(4 / r_{c}^{2}\right)\left(c_{P} / c_{V}\right)\left(k T_{e} / m_{e}\right),
$$

which describes the frequency of an electron plasma wave with wave vector $k=2 / r_{c}$. The temperature-dependent term is a small correction when $\lambda_{\mathrm{D} e} \ll r_{c}$. The parameters $a_{0}$ and $\theta$, which describe the amplitude and phase of the electron plasma oscillations (i.e., betatron oscillations), depend upon the initial conditions (i.e., beam injection parameters) of the problem.

The sharp resonance in Eq. (6) results from the use of fluid equations with a scalar pressure gradient term, whose magnitude was estimated by assuming that the $e$ beam contracts and expands without changing shape. This results in a neglect of kinetic (Landau) damping and phase-mix damping. In order to include the effects of damping while using fluid equations, we add a phenomenological damping term, $-2 \alpha(d \delta / d t)$, to the right-hand side of Eq. (5), obtaining the equation of a driven damped harmonic oscillator with solution

$$
\begin{aligned}
r(t)= & r_{0}+a_{0} \exp (-\alpha t) \cos \left[\left(\omega_{0}^{2}-\alpha^{2}\right)^{1 / 2} t+\theta\right] \\
& +\frac{e^{2} \int_{0}^{r_{0}} n_{1}\left(r^{\prime}\right) 2 \pi r^{\prime} d r^{\prime}}{2 \pi \epsilon_{0} m_{e} r_{0}} \\
& \times \operatorname{Re}\left[\left(\frac{1}{\omega^{2}-\omega_{0}^{2}+2 i \alpha \omega}\right) \exp (-i \omega t)\right] .
\end{aligned}
$$

For the case of a cold beam $\left(T_{e}=0\right)$ with a flat ion channel profile $\left[n_{0}(r) \equiv n_{0}, n_{1}(r) \equiv n_{1}\right.$, for $\left.r<r_{\text {beam }}\right]$, Eq. (8) becomes

$$
\begin{aligned}
r(t)= & r_{0}+a_{0} \exp (-\alpha t) \cos \left[\left(\omega_{0}^{2}-\alpha^{2}\right)^{1 / 2} t+\theta\right] \\
& +\frac{n_{1}}{2 n_{0}} r_{0} \operatorname{Re}\left[\left(\frac{\omega_{0}^{2}}{\omega^{2}-\omega_{0}^{2}+2 i \alpha \omega}\right) \exp (-i \omega t)\right] .
\end{aligned}
$$

From Eqs. (8) and (9), we note that the damping constant $\alpha$ 
is the imaginary part of the complex electron oscillation frequency. Damping of electron oscillations can arise from kinetic effects such as Landau damping. Since the oscillation frequency of fluid elements depends upon the local ion density, neighboring fluid elements will tend to oscillate at different frequencies. Because the fluid elements are coupled by the pressure gradient when $T_{e} \neq 0$, this can result in phasemix damping of the betatron oscillations, further increasing the value of $\alpha$. A possible estimate of the magnitude of kinetic damping effects is given by the Landau damping constant $\alpha_{\mathrm{L}}$, which depends upon the wave vector of the plasma oscillations ${ }^{6}$ :

$\alpha_{\mathrm{L}}=\omega_{p e}(\pi / 8)^{1 / 2} e^{-3 / 2}\left(k \lambda_{\mathrm{D} e}\right)^{-3} \exp \left(-1 / 2 k^{2} \lambda_{\mathrm{D} e}^{2}\right)$.

A reasonable value of $k$ that can be inserted into Eq. (10) is $k=2 / r_{c}$, which earlier was shown to give agreement between the frequency of oscillation of the fluid elements and the dispersion relation describing electron plasma waves in a spatially uniform plasma.

\section{B. Discussion of radial oscillations}

With $n_{1}(r) \equiv 0$ (uniform ion channel density), the solution (8) reduces to

$r(t)=r_{0}+a_{0} \exp (-\alpha t) \cos \left[\left(\omega_{0}^{2}-\alpha^{2}\right)^{1 / 2} t+\theta\right]$,

which describes damped betatron oscillations of the $e$-beam electrons. The case where $a_{0}=0$ describes force-free propagation. For a cool electron beam, force-free propagation requires that $n_{e} \approx n_{0}$ (in the $e$-beam frame). The condition $n_{e} \approx n_{0}$ reduces to the conventional expression $n_{e_{\mathrm{lab}}} \approx \gamma^{2} n_{i_{\mathrm{abb}}}$ in the laboratory frame.

In the laboratory frame, the oscillations described by Eq. (11) appear as stationary betatron oscillations. For a cool beam, such that the electron Debye length (in the electron frame) is much smaller than the beam radius, the betatron wavelength is

$$
\lambda_{\text {lab }}=2 \pi \beta c\left(\epsilon_{0} m_{e} \gamma / n_{i_{\mathrm{lab}}} e^{2}\right)^{1 / 2} .
$$

For a Gaussian beam and ion channel of equal RMS radii $r_{c}$, the beam-averaged betatron wavelength is

$$
\left\langle\lambda_{\mathrm{lab}}\right\rangle=4 \pi \beta c\left(\epsilon_{0} m_{e} \gamma / n_{0 \mathrm{ab}} e^{2}\right)^{1 / 2},
$$

where $n_{0_{\text {lab }}}$ is the ion density in the center of the ion channel, measured in the lab frame. The beam-averaged betatron wavelength is twice the value of the betatron wavelength describing the center of the beam. For $\beta \sim 1, \mathrm{Eq}$. (13) equals the conventional expression ${ }^{1}\left[2 \pi r_{c}\left(I_{\mathrm{A}} / f I_{b}\right)^{1 / 2}\right]$ for the betatron wavelength, where $f$ is the charge neutralization fraction, $I_{b}$ is the beam current, and $I_{\mathrm{A}}$ is the Alfvén current.

When $n_{1}(r)$ is nonzero, there are additional oscillations of the $e$-beam radius at the frequency $\omega$ of the ion density oscillations, as seen in the frame moving with the $e$ beam. A resonant excitation occurs when the frequency $\omega$ equals the natural frequency $\omega_{0}$ of the betatron oscillations, and large oscillations of the beam electrons occur for small variations in ion density when $\omega \approx \omega_{0}$. In the laboratory frame, the resonance condition occurs when the wavelength of the ion density variation equals the betatron wavelength.

In order that the solution ( 8 ) be consistent with the assumption that $\delta \ll r_{0}$ (i.e., the oscillations in beam radius are relatively small), two conditions must be satisfied.

(a) $a_{0} \ll r_{0}$. This condition requires that the magnitude of the betatron oscillations must be small, which can be ensured by matching the beam radius to the ion channel density.

$$
\text { (b) }\left|\frac{e^{2} \int n_{1}\left(r^{\prime}\right) 2 \pi r^{\prime} d r^{\prime}}{2 \pi r_{0}^{2} \epsilon_{0} m_{e}\left(\omega^{2}-\omega_{0}^{2}+2 i \alpha \omega\right)}\right| \ll 1 \text {. }
$$

This condition limits the magnitude of the driving force, which results from the ion density variations. For a cold beam with a flat ion channel profile in which the ion density periodically drops to zero, $n_{0}(r)=n_{1}(r) \equiv n$ (treated by Miller and Gilgenbach ${ }^{3}$ ), this condition reduces to $\frac{1}{\mid} \mid \omega_{0}^{2} /$ $\left(\omega^{2}-\omega_{0}^{2}+2 i \alpha \omega\right) \mid \ll 1$ [see Eq. (9)], which can only be satisfied when $\omega>\omega_{0}$, i.e., the wavelength of ion density variations must be short compared to the betatron wavelength.

In summary, these calculations indicate that the electrons in an $e$ beam propagating through a stationary periodic ion density channel, with no external magnetic field, will undergo cylindrically symmetric oscillations at their betatron wavelength as well as the wavelength of the ion density variation. These oscillations result in a modulation of the electron density. When the wavelength of the ion density variation is near the betatron wavelength, resonant excitation results in large magnitude radial oscillations of the beam electrons, even if the relative variation in ion density is small.

\section{IFR HOSE INSTABILITY}

The propagation of an $e$ beam in an IFR ion density channel can be disrupted by the ion hose (i.e., ion resonance) instability, ${ }^{7-9}$ in which transverse oscillations of the $e$ beam and ion channel couple and grow. Since this instability involves resonant excitation of ion and electron plasma oscillations, increased stability may be provided by the axial variation of the ion and electron densities resulting from a periodic ion channel.

In order to examine this possibility, we consider the rigid beam treatment of the ion hose instability for wavelengths long compared to the beam radius. In this approach, the $E$ field that results from transverse displacements is calculated by assuming that the cross-sectional density profiles of the $e$ beam and ion channel remain constant. We calculate in the laboratory frame of reference, where the electron beam propagates in an ion channel parallel to an applied magnetic field $B_{0} z$. (The case where $B_{0}=0$ is included.)

Letting $\left(X_{i}(z, t), Y_{i}(z, t)\right)$ and $\left(X_{e}(z, t), Y_{e}(z, t)\right)$ denote the transverse coordinates of the ion channel and $e$ beam, respectively, the electric field at the center of the displaced ion beam is

$$
\left(E_{x}, E_{y}\right)=\left(-n_{e} e / 2 \epsilon_{0}\right)\left(X_{i}-X_{e}, Y_{i}-Y_{e}\right),
$$

where $n_{e}$ is the electron density in the center of the $e$ beam. A similar formula relates the electric field at the center of the $e$ beam to the ion density $n_{i}$ in the center of the ion channel. Thus the fluid equations for the center of the ion channel and electron beam are 


$$
\begin{aligned}
\frac{d^{2} X_{i}}{d t^{2}}= & -\left(\frac{n_{e} e^{2}}{2 \epsilon_{0} m_{i}}\right)\left(X_{i}-X_{e}\right)+\omega_{c i} \frac{d Y_{i}}{d t}-2 \alpha_{i} \frac{d X_{i}}{d t}, \\
\frac{d^{2} Y_{i}}{d t^{2}}= & -\left(\frac{n_{e} e^{2}}{2 \epsilon_{0} m_{i}}\right)\left(Y_{i}-Y_{e}\right)-\omega_{c i} \frac{d X_{i}}{d t}-2 \alpha_{i} \frac{d Y_{i}}{d t}, \\
\frac{d^{2} X_{e}}{d t^{2}}= & -\left(\frac{n_{i} e^{2}}{2 \epsilon_{0} \gamma m_{e}}\right)\left(X_{e}-X_{i}\right) \\
& -\omega_{c e} \frac{d Y_{e}}{d t}-2 \alpha_{e} \frac{d X_{e}}{d t} \\
\frac{d^{2} Y_{e}}{d t^{2}=} & -\left(\frac{n_{i} e^{2}}{2 \epsilon_{0} \gamma m_{e}}\right)\left(Y_{e}-Y_{i}\right) \\
& +\omega_{c e} \frac{d X_{e}}{d t}-2 \alpha_{e} \frac{d Y_{e}}{d t} .
\end{aligned}
$$

In these equations, $\omega_{c i}$ and $\omega_{c e}$ are the cyclotron frequencies $\left(e B_{0} / m_{i}\right.$ and $e B_{0} / \gamma m_{e}$, respectively, with $e>0$ ), while $\alpha_{i}$ and $\alpha_{e}$ are phenomenological damping constants in order to account for kinetic effects such as Landau damping and phase-mix damping. Setting $Z_{i}=X_{i}+i Y_{i}, Z_{e}=X_{e}+i Y_{e}$, and defining the frequencies $\omega_{i} \equiv\left(n_{e} e^{2} / 2 \epsilon_{0} m_{i}\right)^{1 / 2}, \omega_{e}$ $\equiv\left(n_{i} e^{2} / 2 \epsilon_{0} \gamma m_{e}\right)^{1 / 2}$, we can write Eq. (14) as

$$
\begin{aligned}
& \frac{d^{2} Z_{i}}{d t^{2}}=-\omega_{i}^{2}\left(Z_{i}-Z_{e}\right)-\left(2 \alpha_{i}+i \omega_{c i}\right) \frac{d Z_{i}}{d t}, \\
& \frac{d^{2} Z_{e}}{d t^{2}}=-\omega_{e}^{2}\left(Z_{e}-Z_{i}\right)-\left(2 \alpha_{e}-i \omega_{c e}\right) \frac{d Z_{e}}{d t} .
\end{aligned}
$$

We note that $\omega_{e}$ is $2^{-1 / 2}$ times the betatron frequency describing the center of the beam. Equations similar to (15) can also be calculated for off-axis fluid elements, again using the rigid beam formalism. Because of the radial variation in the $e$ beam and ion channel densities, the frequencies $\omega_{i}$ and $\omega_{e}$ are different for different fluid elements, and additional terms occur since the $E$ fields are not always parallel to the displacement for off-axis fluid elements. Clearly, the tendency of different fluid elements to oscillate at different frequencies and in different directions will cause a change in the density profiles of the ion channel and $e$ beam, in violation of the rigid beam assumption. This effect will be discussed later.

First, we consider the case where the beam and channel are rigid and there is no axial variation in electron and ion density so that $\omega_{i}^{2}$ and $\omega_{e}^{2}$ and constants. For solutions of the form $Z_{e}(z, t)=Z_{e 0} e^{i(k z-\omega t)}, Z_{i}(z, t)=Z_{00} e^{i(k z-\omega t)}$, where $\omega$ is real, we utilize the convective derivative $d / d t$ $=\partial / \partial t+v \partial / \partial z$ for the $e$ beam, and obtain

$$
\begin{aligned}
& -\omega^{2} Z_{i}=-\omega_{i}^{2}\left(Z_{i}-Z_{e}\right)-\left(2 \alpha_{i}+i \omega_{c i}\right)\left(-i \omega Z_{i}\right), \\
& -\Omega^{2} Z_{e}=-\omega_{e}^{2}\left(Z_{e}-Z_{i}\right)-\left(2 \alpha_{e}-i \omega_{c e}\right)\left(-i \Omega Z_{e}\right),
\end{aligned}
$$

where $\Omega=\omega-v k$. Eliminating the quantities $Z_{e}$ and $Z_{i}$ yields

$\mathbf{\Omega}^{2}+2\left(i \alpha_{e}+\frac{\omega_{c e}}{2}\right) \mathbf{\Omega}=\frac{\omega_{e}^{2}\left(\omega^{2}-\omega \omega_{c i}+2 i \omega \alpha_{i}\right)}{\omega^{2}-\omega \omega_{c i}+2 i \omega \alpha_{i}-\omega_{i}^{2}}$.

Using the quadratic formula to solve for $\Omega$, we obtain the dispersion relation

$$
\begin{aligned}
k=\frac{\omega-\Omega}{v}= & \frac{1}{v}\left\{\omega+\frac{\omega_{c e}}{2}+i \alpha_{e} \pm\left[\left(\omega_{c e}+i \alpha_{e}\right)^{2}\right.\right. \\
& \left.\left.+\omega_{e}^{2}\left(\frac{\omega^{2}-\omega \omega_{c i}+2 i \omega \alpha_{i}}{\omega^{2}-\omega \omega_{c i}+2 i \omega \alpha_{i}-\omega_{i}^{2}}\right)\right]^{1 / 2}\right\}
\end{aligned}
$$

In the case where $\omega \operatorname{Re}(k)>0$ and $\operatorname{Im}(k)<0$, Eq. (18) describes transverse (hose) oscillations of the beam that propagate downstream and grow.

We note that, for the case of no magnetic field $\left(\omega_{c i}=\omega_{c e}=0\right)$ and weak damping $\left(\alpha_{i} \ll \omega_{i}, \alpha_{e} \ll \omega_{e}\right)$, Eq. (18) is identical to the dispersion relation describing longitudinal oscillations of electrons flowing through ions. This was obtained for the boundary value problem (where $\omega$ is real) using the Vlasov picture with Cauchy velocity distributions $\left(F_{0 e}\left(v_{z}\right)=C_{e} / \pi\left[C_{e}^{2}+\left(v_{z}-v\right)^{2}\right], \quad F_{0 i}\left(v_{z}\right)=C_{i} /\right.$ $\pi\left[C_{i}^{2}+v_{z}^{2}\right]$ ), where $\omega_{e}$ is the electron plasma frequency, $\omega_{i}$ is the ion plasma frequency, $\alpha_{i}=(\omega /|\omega|) C_{i} k$, and $\alpha_{e}=(\omega /|\omega|) C_{e} k$. This supports the use of phenomenological damping constants $\left(\alpha_{i}, \alpha_{e}>0\right)$ as a reasonable way to account for the kinetic effects of Landau damping on the ion hose waves propagating downstream, for which $\omega \operatorname{Re}(k)>0$.

Resonant excitation of the hose instability occurs when the magnitude of the denominator on the rhs of Eq. (18) is minimized, which requires

$$
\omega=\omega_{r}=\left(\omega_{c i} / 2\right) \pm\left(\omega_{i}^{2}+\omega_{\varepsilon i}^{2} / 4\right)^{1 / 2} .
$$

In the absence of an axial magnetic field, the resonant frequencies are $\pm \omega_{i}$.

Provided that the ion damping constant is sufficiently small that $\left|\alpha_{i} \omega_{r} / \omega_{i}^{2}\right| \ll 1$, and the axial magnetic field is suffciently weak that

$$
\left|\alpha_{i} \omega_{r} / \omega_{i}^{2}\right|\left|i \alpha_{e}+\omega_{c e} / 2\right|^{2} \ll \omega_{e}^{2},
$$

the value of $k$ for $\omega=\omega_{r}$ is dominated by the resonant term, yielding

$$
k= \pm \frac{\omega_{e}}{v}\left|\frac{\omega_{i}^{2}}{\omega_{r} \alpha_{i}}\right|\left(\frac{1-i\left(\omega_{r} /\left|\omega_{r}\right|\right)}{2}\right) .
$$

This expression describes four modes since there are two resonant frequencies of opposite sign. Two of the four modes describe waves that propagate downstream and grow; these modes obey

$$
|\operatorname{Re}(k)|=-\operatorname{Im}(k)=\left(\omega_{e} / 2 v\right)\left|\omega_{i}^{2} / \omega_{r} \alpha_{i}\right|^{1 / 2} .
$$

Since the $k$ vector for betatron oscillations of the center of the beam is $2^{1 / 2} \omega_{e} / v$ [see Eq. (12)], these modes have wavelength and $e$-folding length smaller than a betatron wavelength for $\left|\alpha_{i} \omega_{r} / \omega_{i}^{2}\right|<1$.

In the limit $\alpha_{i} \rightarrow 0$, Eq. (21) indicates that the value of $k$ for $\omega=\omega_{r}$ diverges so that an absolute instability occurs in the absence of ion damping. This deficiency of the rigid beam approximation in the limit of weak ion damping results from the assumption that $\omega_{e}^{2}$ and $\omega_{i}^{2}$ are constant in Eq. (15). In a propagating beam, longitudinal (compressional) wave modes are unstable; in addition, betatron oscillations and turbulence will be present. The resultant variations in the electron and ion densities cause a variation in the values of 
$\omega_{e}^{2}$ and $\omega_{i}^{2}$ since $\omega_{e}^{2} \propto n_{i}$ and $\omega_{i}^{2} \propto n_{e}$. In addition, deformations of the $e$ beam and ion channel that are associated with ion hose oscillations cause a change of the electron and ion densities from those assumed in the rigid beam approximation. As a result, the nonrigidity of the electron beam and ion channel will also lead to variations in the terms $\omega_{e}^{2}$ and $\omega_{i}^{2}$, which appear in Eq. (15).

In order to study the effects of variations in $\omega_{e}^{2}$ and $\omega_{i}^{2}$ resulting from axial density variations and the nonrigidity of the beam and channel, we consider Eq. (15), letting $\omega_{e}^{2}$ and $\omega_{i}^{2}$ be functions of $z$ and $t$. Since the use of a periodic ion channel will result in axial variations in the electron and ion densities, the following results include the case of periodic ion channels. With the definitions

$$
\begin{aligned}
& \Omega^{2} \equiv-Z_{e}^{-1} \frac{d^{2} Z_{e}}{d t^{2}}, \quad \Omega \equiv i Z_{e}^{-1} \frac{d Z_{e}}{d t}, \\
& \omega^{2} \equiv-Z_{i}^{-1} \frac{d^{2} Z_{i}}{d t^{2}}, \quad \omega \equiv i Z_{i}^{-1} \frac{d Z_{i}}{d t},
\end{aligned}
$$

Eqs. (16) and (17) then follow, in which $\Omega^{2}, \Omega, \omega^{2}, \omega$, $\omega_{i}^{2}$, and $\omega_{e}^{2}$ now represent functions of $z$ and $t$.

We assume that variations in $\omega_{e}^{2}$ and $\omega_{i}^{2}$ are distributed so that the averages of functions of $\omega_{e}^{2}$ and $\omega_{i}^{2}$ over the region $[z, z+\delta z] \times[t, t+\delta t]$ are independent of $z$ and $t$ for some value of $\delta z$ and $\delta t$. This will be obeyed, e.g., by random variations and periodic variations. We denote such averages by $\langle\cdots\rangle$. Averaging Eq. (17) yields

$$
\left\langle\Omega^{2}\right\rangle+2\left(i \alpha_{e}+\frac{\omega_{c e}}{2}\right)\langle\Omega\rangle=\left\langle\frac{\omega_{e}^{2}\left(\omega^{2}-\omega \omega_{c i}+2 i \omega \alpha_{i}\right)}{\omega^{2}-\omega \omega_{c i}+2 i \omega \alpha_{i}-\omega_{i}^{2}}\right\rangle,
$$

where $\Omega^{2}, \Omega, \omega^{2}, \omega, \omega_{i}^{2}$, and $\omega_{e}^{2}$ are functions of $z$ and $t$.

In order to proceed further, we assume the existence of solutions to Eq. (22) that describe coherent sinusoidal oscillations, i.e.,

$$
Z_{e}(z, t) \approx Z_{e 0} e^{i(k z-\omega t)}, \quad Z_{i}(z, t) \approx Z_{i 0} e^{i(k z-\omega t)},
$$

where $\omega$ is a real constant. Exact equality will not be obeyed because of the variations in $\omega_{i}^{2}$ and $\omega_{e}^{2}$. Because of the large mass of the ions, we assume that the level of variations in the ion channel motion will be relatively small, so that $\omega^{2}(z, t) \equiv \omega^{2}$ and $\omega(z, t) \equiv \omega$ will be approximately obeyed. For solutions of this form, $\left\langle\Omega^{2}(z, t)\right\rangle \approx(\omega-v k)^{2}$ and $\langle\Omega(z, t)\rangle \approx \omega-v k$ will be satisfied. With these substitutions, Eq. (22) can be solved for $k$ to obtain a dispersion relation

$$
\begin{aligned}
k= & \frac{1}{v}\left\{\omega+\frac{\omega_{c e}}{2}+i \alpha_{e} \pm\left[\left(\frac{\omega_{c e}}{2}+i \alpha_{e}\right)^{2}\right.\right. \\
& \left.\left.+\left(\frac{\omega_{e}^{2}\left(\omega^{2}-\omega \omega_{c i}+2 i \omega \alpha_{i}\right)}{\omega^{2}-\omega \omega_{c i}+2 i \omega \alpha_{i}-\omega_{i}^{2}}\right)\right]^{1 / 2}\right\},
\end{aligned}
$$

where $\omega_{i}^{2}$ and $\omega_{e}^{2}$ are functions of $z$ and $t$. This dispersion relation reduces to Eq. (18) when $\omega_{i}^{2}$ and $\omega_{e}^{2}$ are constant.

When variations in $\omega_{i}^{2}(z, t)$ are present, the resonance condition $\omega^{2}-\omega \omega_{c i}=\omega_{i}^{2}(z, t)$ can no longer be met for all $z$ and $t$ by any frequency. Consequently, the magnitude of the average in Eq. (23) will be reduced when compared to its value at resonance when the variations in $\omega_{i}^{2}(z, t)$ were disregarded [Eq. (18) ], resulting in a reduction of the magnitude of $k$. This leads to an increase in the $e$-folding length of the ion hose instability, i.e., a "detuning" of the resonance.

We recall that $\omega_{i}^{2} \approx n_{e} e^{2} / 2 \epsilon_{0} \gamma m_{e}$, so that variations in $\omega_{i}^{2}$ result from variations in the electron density on axis as well as the nonrigidity of the beam and channel. The resonance detuning that results from the nonrigidity of the beam and channel has been previously observed in spread-mass models. ${ }^{8}$

In order to evaluate Eq. (23), we approximate

$$
\begin{aligned}
\left\langle\frac{\omega_{e}^{2}\left(\omega^{2}-\omega \omega_{c i}+2 i \omega \alpha_{i}\right)}{\omega^{2}-\omega \omega_{c i}+2 i \omega \alpha_{i}-\omega_{i}^{2}}\right) & \\
\approx & \left(\omega_{e}^{2}\right\rangle\left(\omega^{2}-\omega \omega_{c i}+2 i \omega \alpha_{i}\right) \\
\quad & \times\left\langle\frac{1}{\omega^{2}-\omega \omega_{c i}+2 i \omega \alpha_{i}-\omega_{i}^{2}}\right\rangle
\end{aligned}
$$

In evaluating the average of Eq. (24), we note that we can write

$$
\begin{aligned}
I & \equiv\left\langle\frac{1}{\omega^{2}-\omega \omega_{c i}+2 i \omega \alpha_{i}-\omega_{i}^{2}}\right\rangle \\
& =\int_{-\infty}^{\infty} \frac{f\left(\omega_{i}^{2}\right) d\left(\omega_{i}^{2}\right)}{\omega^{2}-\omega \omega_{c i}+2 i \omega \alpha_{i}-\omega_{i}^{2}},
\end{aligned}
$$

where $f\left(\omega_{i}^{2}\right)$ is the distribution of $\omega_{i}^{2}(z, t)$ in the region $[z, z+\delta z] \times[t, t+\delta t]$, normalized so that $\int f\left(\omega_{i}^{2}\right)$ $\times d\left(\omega_{i}^{2}\right)=1$. We allow the possibility that $f\left(\omega_{i}^{2}\right)>0$ for $\omega_{i}^{2}<0$, which describes a repulsive force on the center of the ion channel. This could easily occur if the ion channel and electron beam profiles are deformed.

In order to determine the behavior of Eq. (25) for a distribution peaked at $\left\langle\omega_{i}^{2}\right\rangle$ with half-width $\delta \omega_{i}^{2}$, we consider a Gaussian distribution

$$
\begin{aligned}
f\left(\omega_{i}^{2}\right)= & {\left[(2 \pi)^{1 / 2} \delta \omega_{i}^{2}\right]^{-1} } \\
& \times \exp \left\{-(1 / 2)\left[\left(\omega_{i}^{2}-\left\langle\omega_{i}^{2}\right\rangle\right) / \delta \omega_{i}^{2}\right]^{2}\right\} .
\end{aligned}
$$

In this case,

$$
\begin{aligned}
I= & -\left(2^{1 / 2} \delta \omega_{i}^{2}\right)^{-1} \\
& \times Z\left[\left(\omega^{2}-\omega \omega_{c i}-\left\langle\omega_{i}^{2}\right\rangle+2 i \omega \alpha_{i}\right) / 2^{1 / 2} \delta \omega_{i}^{2}\right],
\end{aligned}
$$

where, for $\omega>0, Z$ is the plasma dispersion function, while for $\omega<0, Z$ is the complex conjugate of the plasma dispersion function. From the properties of the plasma dispersion function, ${ }^{10}$ we observe that $|I|$ and $|\operatorname{Im}(I)|$ are maximized for frequencies that satisfy

$$
\left|\omega^{2}-\omega \omega_{c i}-\left\langle\omega_{i}^{2}\right\rangle\right|<\left|2 \omega \alpha_{i}\right|+2^{1 / 2} \delta \omega_{i}^{2},
$$

i.e., $\omega^{2}-\omega \omega_{c i} \approx\left\langle\omega_{i}^{2}\right\rangle$. For these resonant frequencies, the large values of $|I|$ and $|\operatorname{Im}(I)|$ result in the minimum $e$ folding length for ion hose oscillations, when substituted in the dispersion relation (23). Thus the detuning of the ion hose resonance that results from variations in $\omega_{i}^{2}(z, t)$ does not greatly change the resonant frequencies, but only the wavelength and $e$-folding length of the corresponding modes.

For the resonant frequencies where $\omega^{2}-\omega \omega_{c i} \approx\left\langle\omega_{i}^{2}\right\rangle$, $|\operatorname{Re}(I)| \ll|\operatorname{Im}(I)|$, so that 


$$
\begin{aligned}
I \approx & -i \frac{\omega}{|\omega|}\left(\frac{\pi}{2}\right)^{1 / 2} \frac{1}{\delta \omega_{i}^{2}} \\
& \times\left[\exp \left(\frac{2^{1 / 2} \omega \alpha_{i}}{\delta \omega_{i}^{2}}\right)^{2}\left(1-\operatorname{erf}\left|\frac{2^{1 / 2} \omega \alpha_{i}}{\delta \omega_{i}^{2}}\right|\right)\right]
\end{aligned}
$$

Substituting Eq. (26) into the dispersion relation (23), we obtain the value of $k$ for the resonant modes, which have the smallest $e$-folding lengths,

$$
\begin{aligned}
k= & \frac{1}{v}\left(\omega+\frac{\omega_{c e}}{2}+i \alpha_{e} \pm\left\{\left(\frac{\omega_{c e}}{2}+i \alpha_{e}\right)^{2}\right.\right. \\
& -\left\langle\omega_{e}^{2}\right\rangle \frac{i \omega}{|\omega|}\left(\frac{\pi}{2}\right)^{1 / 2} \frac{\left\langle\omega_{i}^{2}\right\rangle+2 i \omega \alpha_{i}}{\delta \omega_{i}^{2}} \\
& \left.\left.\times\left[\exp \left(\frac{2^{1 / 2} \omega \alpha_{i}}{\delta \omega_{i}^{2}}\right)^{2}\left(1-\operatorname{erf}\left|\frac{2^{1 / 2} \omega \alpha_{i}}{\delta \omega_{i}^{2}}\right|\right)\right]\right\}^{1 / 2}\right) .
\end{aligned}
$$

In the limit of small ion damping $\left(\alpha_{i} \rightarrow 0\right)$, Eq. (27) becomes

$$
\begin{aligned}
k= & \frac{1}{v}\left\{\omega+\frac{\omega_{c e}}{2}+i \alpha_{e}\right. \\
& \left. \pm\left[\left(\frac{\omega_{c e}}{2}+i \alpha_{e}\right)^{2}-\left\langle\omega_{e}^{2}\right\rangle \frac{i \omega}{|\omega|}\left(\frac{\pi}{2}\right)^{1 / 2} \frac{\left\langle\omega_{i}^{2}\right\rangle}{\delta \omega_{i}^{2}}\right]^{1 / 2}\right\} .
\end{aligned}
$$

We recall that, for variations in $\omega_{i}^{2}(z, t)$ caused by variations in the electron density, $\delta \omega_{i}^{2} /\left\langle\omega_{i}^{2}\right\rangle \approx \delta n_{e} / n_{e}$. This term is of order 1 in a beam with moderate variations in electron density, which can arise from turbulence, longitudinal oscillations, the use of a periodic ion channel, and the nonrigidity of the beam and channel.

A comparison of Eq. (28) with Eq. (18) (evaluated at resonance) indicates that the resonance detuning resulting from variations in $\omega_{i}^{2}(z, t)$ is comparable to that resulting from an ion damping term $\alpha_{i}$, provided that $\alpha_{i} / \omega_{i}$ $\approx(2 \pi)^{-1 / 2} \delta \omega_{i}^{2} /\left\langle\omega_{i}^{2}\right\rangle$. Thus, for weak ion damping $\left[\alpha_{i} / \omega_{i}<(2 \pi)^{-1 / 2} \delta \omega_{i}^{2} /\left\langle\omega_{i}^{2}\right\rangle\right]$, the variations in $\omega_{i}^{2}(z, t)$ are an important source of resonance detuning.

For a strong axial magnetic field, such that $\left.\omega_{c e}^{2}\right\rangle\left\langle\omega_{e}^{2}\right\rangle$ $\times\left(\left\langle\omega_{i}^{2}\right\rangle / \delta \omega_{i}^{2}\right)^{2}$, and negligible damping $\left(\alpha_{i} \rightarrow 0, \alpha_{e} \rightarrow 0\right)$, Eq. (28) reduces to

$k=\frac{1}{v}\left\{\omega+\frac{\omega_{c e}}{2} \pm\left[\frac{\omega_{c e}}{2}-i \frac{\omega}{|\omega|}\left(\frac{\pi}{2}\right)^{1 / 2} \frac{\left\langle\omega_{e}^{2}\right\rangle}{\omega_{c e}} \frac{\left\langle\omega_{i}^{2}\right\rangle}{\delta \omega_{i}^{2}}\right]\right\}$.

This equation describes growing oscillations with

$$
-\operatorname{Im}(k)=\frac{\left(2\left\langle\omega_{e}^{2}\right\rangle\right)^{1 / 2}}{v} \frac{\left(\left\langle\omega_{e}^{2}\right\rangle\right)^{1 / 2}}{\left|\omega_{c e}\right|} \frac{\left\langle\omega_{i}^{2}\right\rangle}{\delta \omega_{i}^{2}} \frac{\pi^{1 / 2}}{2} .
$$

Since $\left(2\left\langle\omega_{e}^{2}\right\rangle\right)^{1 / 2} / v$ is the wave vector of a betatron oscillation, the $e$-folding length with a strong axial magnetic field, negligible damping, and moderate density variations, is many betatron wavelengths.

For the case of a weak axial magnetic field or no magnetic field $\left(\omega_{c e}^{2} \ll\left\langle\omega_{e}^{2}\right\rangle\left\langle\omega_{i}^{2}\right\rangle / \delta \omega_{i}^{2}\right)$ and negligible damping, the value of the wave vector at resonance is again found using Eq. (28):

$$
\begin{aligned}
k= & \frac{1}{v}\left[\omega+\frac{\omega_{c e}}{2}\right. \\
& \left. \pm\left\langle\omega_{e}^{2}\right\rangle^{1 / 2}\left(\frac{\pi}{8}\right)^{1 / 4}\left(\frac{\left\langle\omega_{i}^{2}\right\rangle}{\delta \omega_{i}^{2}}\right)^{1 / 2}\left(1-i \frac{\omega}{|\omega|}\right)\right] .
\end{aligned}
$$

The modes that propagate downstream obey

$$
|\operatorname{Re}(k)|=-\operatorname{Im}(k)=\frac{\left(2\left\langle\omega_{e}^{2}\right\rangle\right)^{1 / 2}}{v}\left(\frac{\pi}{32}\right)^{1 / 4}\left(\frac{\left\langle\omega_{i}^{2}\right\rangle}{\delta \omega_{i}^{2}}\right)^{1 / 2}
$$

Thus for moderate variations in beam density or deformations of the beam and channel so that $\delta \omega_{i}^{2} /\left(\omega_{i}^{2}\right\rangle \approx 1$, and negligible damping, the wavelength and $e$-folding length are approximately equal to two betatron wavelengths. In the case of a Gaussian beam and channel, this is equal to the beam-averaged betatron wavelength.

Experimental observations have shown that transverse oscillations of an electron beam occur after propagation distances on the order of a betatron wavelength in an IFR channel with no axial magnetic field, ${ }^{9}$ consistent with the predictions of this model. Numerical calculations with the spread mass model have yielded an $e$-folding length on the order of a betatron wavelength in the absence of an axial magnetic field. ${ }^{8}$ This is also consistent with $\mathrm{Eq}$. (32) since moderate nonrigidity of the beam and channel will lead to values of $\delta \omega_{i}^{2} /\left\langle\omega_{i}^{2}\right\rangle$ on the order of unity.

Since the $e$-folding length of the ion hose instability increases with $\delta \omega_{i}^{2} /\left\langle\omega_{i}^{2}\right\rangle$, which increases with $\delta n_{e} / n_{e}$, increasing the axial variation of electron density will increase the $e$-folding length of the ion hose instability. This suggests that the use of periodic ion channels will aid in the propagation of electron beams. In the absence of a magnetic field and damping, our results suggest that the wavelength and $e$-folding length can be increased to approximately the beam-averaged betatron wavelength.

\section{SUMMARY AND CONCLUSIONS}

The results of this investigation of the propagation of an electron beam in a periodic ion density channel can be summarized as follows.

(1) In the absence of an external magnetic field, the electrons undergo cylindrically symmetric radial oscillations at their betatron wavelength as well as the wavelength of the ion density variation. The betatron oscillations appear as electron plasma oscillations in the frame of reference moving with the $e$ beam. When the wavelength of the ion density variation is equal to the betatron wavelength, resonant excitation results in large radial oscillations of the electrons even if the relative variation in ion density is small. In the frame of reference moving with the $e$ beam, this appears as resonant excitation of electron oscillations when the driving frequency equals the plasma frequency.

(2) A dispersion relation for IFR ion hose oscillations was obtained in which electron and ion density variations (including the nonrigidity of the electron beam and ion channel) result in the detuning of the ion hose resonance. Larger axial variations in electron density (resulting, e.g., from the use of a periodic ion density channel) increase the 
$e$-folding length of the hose oscillation. In the absence of a magnetic field and significant damping of electron or ion oscillations, moderate density variations (caused by, e.g., a periodic ion channel, turbulence, or the nonrigidity of the beam and channel) result in a wavelength and $e$-folding length on the order of the beam-averaged betatron wavelength.

\section{ACKNOWLEDGMENTS}

This research was supported by the Office of Naval Research, National Science Foundation Grant No. ECS8351837, and SDIO-IST.

\section{APPENDIX: MAGNETIC FORCES}

The consistency of the solution obtained in Sec. II for the radial electron oscillations in a periodic ion channel requires that the magnetic forces be negligible in the frame of reference moving with the beam. We check this for a fluid element on the edge of a cool $e$ beam of uniform cross section $\left[n_{0}(r) \equiv n_{0}\right]$. We obtain an estimate of transverse velocity $\left(v_{t}=d \delta / d t\right)$ from Eq. ( 8$)$ and an estimate of $E$ is obtained by substituting the solution ( 8 ) into Eq. (4). The typical electron velocity resulting from betatron oscillations of magnitude $a_{0}$ is

$$
v_{t} \sim a_{0} \omega_{p e}
$$

The magnetic field strength at the position $r_{0}=r_{\text {beam }}$ due to the ion current density is

$$
B \sim \mu_{0} n_{0} e \beta c r_{0} / 2 .
$$

The magnitude of the electric field generated by betatron oscillations of magnitude $a_{0}$ is

$$
E \sim n_{0} e a_{0} / \epsilon_{0} \text {. }
$$

In order that the magnetic forces be ignorable, we must have $v_{t} B \ll E$. Using the estimates of Eqs. (A1)-(A3), this requirement becomes

$$
a_{0} \omega_{p e} \mu_{0} n_{0} e \beta c r_{0} / 2 \ll n_{0} e a_{0} / \epsilon_{0} .
$$

In terms of the $e$-beam current in the laboratory frame ( $I_{\text {lab }}$ $\left.=\gamma n_{0} e \beta c \pi r_{0}^{2}\right)$, (A4) can be written as

$$
I_{\text {lab }} \ll\left(4 \pi \epsilon_{0} m_{e} c^{3} / e\right)(\gamma / \beta) \text {. }
$$

This consistency condition requires (for $\beta \sim 1$ ) that the electron beam current be less than the Alfvén current limit ${ }^{5}$ $\left[\left(4 \pi \epsilon_{0} m_{e} c^{3} / e\right) \beta \gamma\right]$.

Similarly, for forced oscillations with frequency $\omega$, we obtain the consistency condition

$$
I_{\text {lab }} \ll\left(\frac{4 \pi \epsilon_{0} m_{e} c^{3}}{e}\right)\left(\frac{\gamma}{\beta}\right)\left(\frac{\omega}{\omega_{p e}}\right)^{2} .
$$

This is a more stringent requirement than the Alfvén current limit when $\omega / \omega_{p e} \ll 1$ and $\beta \sim 1$.

For the case where the $e$ beam is propagating parallel to a static magnetic field $B_{0}$, the analysis of Sec. II is consistent provided that $v_{t} B_{0} \ll E$, where the transverse velocity $v_{t}$ results from the betatron and forced oscillations. This requirement is satisfied provided that the electron cyclotron frequency in the frame moving with the $e$ beam $\left(e B_{0} / m_{e}\right)$ is much smaller than $\omega$ and $\omega_{p e}$, which is satisfied only by a weak magnetic field.

'R. L.Carlson, S. W. Downey, and D. C. Moir, J. Appl. Phys. 61, 12 (1987).

${ }^{2}$ R. M. Gilgenbach, R. F. Lucey, Jr., L. D. Horton, M. L. Brake, S. Bidwell, M. Cuneo, J. Miller, and L. Smutek, Bull. Am. Phys. Soc. 30, 1502 (1985); J. D. Miller and R. M. Gilgenbach, ibid. 31, 1428 (1986).

${ }^{3}$ J. D. Miller and R. M. Gilgenbach, Phys. Fluids 30, 3165 (1987).

${ }^{4}$ M. Friedman, V. Serlin, Y. Y. Lau, and J. Krall, Bull. Am. Phys. Soc. 32, 1720 (1987).

${ }^{5}$ H. Alfvén, Phys. Rev. 55, 425 (1939).

${ }^{6}$ D. R. Nicholson, Introduction to Plasma Theory (Wiley, New York, 1983), p. 83.

${ }^{7}$ R. C. Davidson and H. S. Uhm, J. Appl. Phys. 51, 885 (1980).

${ }^{8}$ H. L. Buchanon, Phys. Fluids 30, 221 (1987).

${ }^{9}$ R. F. Schneider and J. R. Smith, Phys. Fluids 29, 3917 (1986).

${ }^{10}$ B. D. Fried and S. D. Conte, The Plasma Dispersion Function (Academic, New York, 1961). 